\title{
Eigenfunctions and fundamental solutions of the fractional Laplace and Dirac operators using Caputo derivatives*
}

\author{
M. Ferreira ${ }^{\dagger \ddagger}, \quad$ N. Vieira ${ }^{\ddagger}$ \\ $\dagger$ School of Technology and Management, \\ Polytechnic Institute of Leiria \\ P-2411-901, Leiria, Portugal. \\ E-mail: milton.ferreira@ipleiria.pt \\ $¥$ CIDMA - Center for Research and Development in Mathematics and Applications \\ Department of Mathematics, University of Aveiro \\ Campus Universitário de Santiago, 3810-193 Aveiro, Portugal. \\ E-mail: mferreira@ua.pt,nloureirovieira@gmail.com
}

\begin{abstract}
In this paper we study eigenfunctions and fundamental solutions for the three parameter fractional Laplace operator ${ }^{C} \Delta_{+}^{(\alpha, \beta, \gamma)}:={ }^{C} D_{x_{0}^{+}}^{1+\alpha}+{ }^{C} D_{y_{0}^{+}}^{1+\beta}+{ }^{C} D_{z_{0}^{+}}^{1+\gamma}$, where $\left.\left.(\alpha, \beta, \gamma) \in\right] 0,1\right]^{3}$ and the fractional derivatives ${ }^{C} D_{x_{0}^{+}}^{1+\alpha},{ }^{C} D_{y_{0}^{+}}^{1+\beta},{ }^{C} D_{z_{0}^{+}}^{1+\gamma}$ are in the Caputo sense. Applying integral transform methods we describe a complete family of eigenfunctions and fundamental solutions of the operator ${ }^{C} \Delta_{+}^{(\alpha, \beta, \gamma)}$ in classes of functions admitting a summable fractional derivative. The solutions are expressed using the Mittag-Leffler function. From the family of fundamental solutions obtained we deduce a family of fundamental solutions of the corresponding fractional Dirac operator, which factorizes the fractional Laplace operator introduced in this paper.
\end{abstract}

Keywords: Fractional partial differential equations; Fractional Laplace and Dirac operators; Caputo derivative; Eigenfunctions; Fundamental solution.

MSC 2010: Primary: 35R11; Secondary: 35A08; 26A33; 30G35; 35P10; 35A22; 35C05.

\section{Introduction}

In the last decades the interest in fractional calculus increased substantially. Among all the subjects there is a considerable interest in the study of ordinary and partial fractional differential equations regarding the mathematical aspects and methods of their solutions and their applications in diverse areas such as physics, chemistry, engineering, optics or quantum mechanics (see, for example [11, 12, 13, 15, 16, 17, 24]).

The fractional Laplace appears e.g. in probabilistic framework as well as in mathematical finance as infinitesimal generators of the stable Lévy processes [1]. One can find also problems involving the fractional Laplace in mechanics and in elastostatics [3] or fluid dynamics [20. Here we propose a fractional Laplace operator in 3-dimensional space using Caputo derivatives with different order for each direction. Previous approaches for this type of operator using Riemann-Liouville derivatives in two and three dimensions can be found in [22] and 7], respectively.

Clifford analysis is a generalization of classical complex analysis in the plane to the case of an arbitrary dimension. At the heart of the theory lies the Dirac operator $D$, a conformally invariant first-order differential

*The final version is published in Complex Variables and Elliptic Equations, 62-No.9, (2017), 1237-1253. It is available via the website https://www.tandfonline.com/doi/full/10.1080/17476933.2016.1250401 
operator which plays the same role as the Cauchy-Riemann operator in complex analysis. In [14, 21] the authors studied the connections between Clifford analysis and fractional calculus, however, the fractional Dirac operator considered in these works do not coincide with the one used here.

The aim of this paper is to present a closed formula for the family of eigenfunctions and fundamental solutions of the three-parameter fractional Laplace operator using Caputo derivatives, as well as a family of fundamental solutions of the associated fractional Dirac operator. For the sake of simplicity we restrict ourselves to the three dimensional case, however the results can be generalized for an arbitrary dimension. The authors would like to point out that the fractional Laplace operator considered in this paper is different from the fractional Laplace operator defined via Fourier transform (see [13]).

The structure of the paper reads as follows: in the Preliminaries we recall some basic facts about fractional calculus and special functions which are necessary for the development of this work. In Section 3 we use operational techniques for the two dimensional Laplace transform and its extension to generalized functions to describe a complete family of eigenfunctions and fundamental solutions of the fractional Laplace operator. In Section 4 we compute a family of fundamental solutions for the fractional Dirac operator. For the particular case of $\alpha=\beta=\gamma=1$ the obtained formulas coincide with the correspondent classical formulas.

\section{Preliminaries}

\subsection{Fractional calculus and special functions}

The left Riemann-Liouville fractional integral of order $\alpha>0$ is given by (see [15])

$$
\left(I_{a^{+}}^{\alpha} f\right)(x)=\frac{1}{\Gamma(\alpha)} \int_{a}^{x} \frac{f(t)}{(x-t)^{1-\alpha}} d t, \quad x>a .
$$

Let $\left({ }^{C} D_{a^{+}}^{\alpha} f\right)(x)$ denote the left fractional Caputo derivative of order $\alpha>0$ on $[a, b] \subset \mathbb{R}$ (see [15])

$$
\left({ }^{C} D_{a^{+}}^{\alpha} f\right)(x):=\left(I_{a^{+}}^{n-\alpha} D^{n} f\right)(x)=\frac{1}{\Gamma(n-\alpha)} \int_{a}^{x} \frac{f^{(n)}(t)}{(x-t)^{1-n+\alpha}} d t, \quad n=[\alpha]+1, \quad x>a,
$$

where $[\alpha]$ means the integer part of $\alpha$. For example, when $0<\alpha<1$ then 22 takes the form

$$
\left({ }^{C} D_{a^{+}}^{\alpha} f\right)(x)=\frac{1}{\Gamma(1-\alpha)} \int_{a}^{x} \frac{f^{\prime}(t)}{(x-t)^{\alpha}} d t .
$$

For the case of the left Riemann-Liouville fractional derivative (here denoted by $\left(D_{a^{+}}^{\alpha} f\right)(x)$ ), the fractional integral and the integer derivative are interchanged, i.e., $\left(D_{a^{+}}^{\alpha} f\right)(x):=\left(D^{n} I_{a^{+}}^{n-\alpha} f\right)(x)$. For more details about the differences between this two fractional derivatives see [15].

We denote by $I_{a^{+}}^{\alpha}\left(L_{1}\right)$ the class of functions $f$ represented by the fractional integral (1) of a summable function, that is $f=I_{a^{+}}^{\alpha} \varphi$, with $\varphi \in L_{1}(a, b)$. A description of this class of functions was given in [18].

Theorem 2.1 A function $f \in I_{a^{+}}^{\alpha}\left(L_{1}\right), \alpha>0$ if and only if $I_{a^{+}}^{n-\alpha} f \in A C^{n}([a, b]), n=[\alpha]+1$ and $\left(I_{a^{+}}^{n-\alpha} f\right)^{(k)}(a)=$ $0, k=0, \ldots, n-1$.

In Theorem 2.1 $A C^{n}([a, b])$ denotes the class of functions $f$ which are continuously differentiable on the segment $[a, b]$ up to order $n-1$ and $f^{(n-1)}$ is absolutely continuous on $[a, b]$. We remark that $\left(I_{a^{+}}^{n-\alpha} f\right)^{(k)}(a)=0$, $k=0, \ldots, n-1$, implies that $f^{(k)}(a)=0, k=0, \ldots, n-1$ (see [17]). Removing the last condition in Theorem 2.1 we obtain the class of functions that admits a summable fractional derivative.

Definition 2.2 ([18]) A function $f \in L_{1}(a, b)$ has a summable fractional derivative $\left(D_{a^{+}}^{\alpha} f\right)(x)$ if $\left(I_{a^{+}}^{n-\alpha} f\right)(x) \in$ $A C^{n}([a, b])$, where $n=[\alpha]+1$.

If a function $f$ admits a summable fractional derivative, then the composition of (2) and (1) can be written in the form (see e.g. [17])

$$
\left(I_{a^{+}}^{\alpha}{ }^{C} D_{a^{+}}^{\alpha} f\right)(x)=f(x)-\sum_{k=0}^{n-1} \frac{f^{(k)}(a)}{k !}(x-a)^{k}, \quad n=[\alpha]+1 .
$$


We remark that if $f \in I_{a^{+}}^{\alpha}\left(L_{1}\right)$ then (4) reduces to $\left(I_{a^{+}}^{\alpha}{ }^{C} D_{a^{+}}^{\alpha} f\right)(x)=f(x)$ due to Theorem 2.1. Nevertheless we note that $\left({ }^{C} D_{a^{+}}^{\alpha} I_{a^{+}}^{\alpha} f\right)(x)=f(x)$ in both cases. It is important to point out that the semigroup property for the composition of Caputo fractional derivatives does not hold in general. We present three sufficient conditions under which the law of exponents hold. They can be applied in different situations accordingly with the conditions assumed to the function $f$.

Theorem 2.3 ([․,$p .56]$ ) Let $f \in C^{k}[a, b], a>0$ and $k \in \mathbb{N}$. Moreover, let $\alpha, \beta>0$ be such that there exists $l \in \mathbb{N}$ with $l \leq k$ and $\alpha, \alpha+\beta \in[l-1, l]$. Then

$$
{ }^{C} D_{a^{+}}^{\alpha}{ }^{C} D_{a^{+}}^{\beta} f(x)={ }^{C} D_{a^{+}}^{\alpha+\beta} f(x) .
$$

This theorem highlights a constraint on the applicability of the semigroup property both with respect to the request of smoothness of the function and with respect to the ranges of the real orders of differentiation $\alpha$ and $\beta$. This means, for example, if $\alpha \in(0,1]$ then the law of exponents is applicable if $\beta \in(0,1-\alpha)$ and $f \in C^{1}[a, b]$, i.e., $f$ and its first derivative are continuous in [a,b] (in $x=a$ and $x=b$ we consider the right and left derivatives, respectively). In most cases the law of exponents is not applicable for fractional Caputo derivatives, but anyhow there are different techniques to handle sequential fractional derivatives (see for example [17]). Since for $f \in C^{[\alpha]+1}[a, b]$ the Caputo derivative is a special case of the Grünwald-Letnikov fractional derivative (see [17, §2.2.3]) then we have the following theorem:

Theorem 2.4 ([17, § 2.2.6]) Let $\alpha, \beta>0$ and $f \in C^{n}([a, b]), a>0, n=[\alpha]+1$. Then

$$
{ }^{C} D_{a^{+}}^{\alpha}{ }^{C} D_{a^{+}}^{\beta} f(x)={ }^{C} D_{a^{+}}^{\alpha+\beta} f(x)
$$

holds for arbitrary $\beta$ if the function $f$ satisfies the conditions

$$
f^{(k)}(a)=0, \quad \text { for } \quad k=0,1, \ldots, n-2 .
$$

For functions $f$ that have a locally integrable singularity at $x=a$ we have the following result.

Theorem 2.5 ([9]) Suppose that $f(x)=(x-a)^{\lambda} g(x)$, where $a, \lambda>0$ and $g(x)$ has the generalized power series expansion $g(x)=\sum_{n=0}^{+\infty} a_{n}(x-a)^{n \gamma}$ with radius of convergence $R>0,0<\gamma \leq 1$. Then

$$
{ }^{C} D_{a^{+}}^{\alpha}{ }^{C} D_{a^{+}}^{\beta} f(x)={ }^{C} D_{a^{+}}^{\alpha+\beta} f(x)
$$

for all $(x-a) \in(0, R)$, the coefficients $a_{n}=0$ for $n$ given by $n \gamma+\lambda-\beta=0$ and either

(a) $\lambda>\mu, \mu=\max (\beta+[\alpha],[\beta+\alpha])$

or

(b) $\lambda \leq \mu, a_{k}=0$, for $k=0,1, \ldots,\left[\frac{\mu-\lambda}{\gamma}\right]$, here $[x]$ denotes the greatest integer less than or equal to $x$.

One important function used in this paper is the two-parameter Mittag-Leffler function $E_{\mu, \nu}(z)$ [10], which is defined in terms of the power series by

$$
E_{\mu, \nu}(z)=\sum_{n=0}^{+\infty} \frac{z^{n}}{\Gamma(\mu n+\nu)}, \quad \mu>0, \nu \in \mathbb{R}, z \in \mathbb{C}
$$

In particular, the function $E_{\mu, \nu}(z)$ is entire of order $\rho=\frac{1}{\mu}$ and type $\sigma=1$. The exponential, trigonometric and hyperbolic functions are expressed through (9) as follows (see [10]):

$$
\begin{gathered}
E_{1,1}(z)=e^{z}, \quad E_{2,1}\left(-z^{2}\right)=\cos (z), \quad E_{2,1}\left(z^{2}\right)=\cosh (z), \\
z E_{2,2}\left(-z^{2}\right)=\sin (z), \quad z E_{2,2}\left(z^{2}\right)=\sinh (z) .
\end{gathered}
$$

From the power series (9) and the operator (1) and (2), we can obtain via straightforward calculations the two following fractional integral and differential formulae involving the two-parametric Mittag-Leffler function:

$$
I_{a^{+}}^{\alpha}\left((x-a)^{\nu-1} E_{\mu, \nu}\left(k(x-a)^{\mu}\right)\right)=(x-a)^{\alpha+\nu-1} E_{\mu, \nu+\alpha}\left(k(x-a)^{\mu}\right)
$$




$$
{ }^{C} D_{a^{+}}^{\alpha}\left((x-a)^{\nu-1} E_{\mu, \nu}\left(k(x-a)^{\mu}\right)\right)=(x-a)^{\nu-\alpha-1} E_{\mu, \nu-\alpha}\left(k(x-a)^{\mu}\right)
$$

for all $\alpha>0, \mu>0, \nu \in \mathbb{R}, k \in \mathbb{C}, a>0, x>a$.

In this paper we make use of the right-sided Laplace transform

$$
F(s)=\int_{T_{f}}^{+\infty} f(t) e^{-s t} d t, \quad T_{f} \geq 0, \quad \operatorname{Re}(s) \geq a_{0}>0
$$

of absolutely integrable functions $f \in L_{1}\left(\left(T_{f},+\infty\right), e^{-a_{0} t} d t\right)$ and its distributional analog $F(s)=\left\langle f(t), e^{-s t}\right\rangle$ in Zemanian's space (see [23]). The formal approach presented in Section 3 leads to the solution of a linear Abel integral equation of the second kind.

Theorem 2.6 ([10, Thm. 4.2]) Let $f \in L_{1}[a, b], \alpha>0$ and $\lambda \in \mathbb{C}$. Then the integral equation

$$
u(x)=f(x)+\frac{\lambda}{\Gamma(\alpha)} \int_{a}^{x}(x-t)^{\alpha-1} u(t) d t, \quad x \in[a, b],
$$

has a unique solution given by

$$
u(x)=f(x)+\lambda \int_{a}^{x}(x-t)^{\alpha-1} E_{\alpha, \alpha}\left(\lambda(x-t)^{\alpha}\right) f(t) d t .
$$

\section{Eigenfunctions and fundamental solution of the fractional Laplace operator}

The aim of this section is to describe a complete family of eigenfunctions and fundamental solutions of the three-parameter fractional Laplace operator. The construction of those functions can be summarized in four steps:

Step 1: Transform the differential equation into an integral equation;

Step 2: Apply the two-dimensional Laplace transform in $y$ and $z$, and obtain a second kind homogeneous integral Volterra equation;

Step 3: Solve the integral equation in $x$;

Step 4: Invert the Laplace transform in $y$ and $z$.

Let us start with the Step 1. Consider the eigenfunction problem for the three-parameter fractional Laplace operator

$$
{ }^{C} \Delta_{+}^{\alpha, \beta, \gamma} u(x, y, z)=\lambda u(x, y, z)
$$

i.e.,

$$
\left({ }^{C} D_{x_{0}^{+}}^{1+\alpha} u\right)(x, y, z)+\left({ }^{C} D_{y_{0}^{+}}^{1+\beta} u\right)(x, y, z)+\left({ }^{C} D_{z_{0}^{+}}^{1+\gamma} u\right)(x, y, z)=\lambda u(x, y, z),
$$

where $\lambda \in \mathbb{C},(\alpha, \beta, \gamma) \in] 0,1]^{3},(x, y, z) \in \Omega=\left[x_{0}, X_{0}\right] \times\left[y_{0}, Y_{0}\right] \times\left[z_{0}, Z_{0}\right], x_{0}, y_{0}, z_{0}>0, X_{0}, Y_{0}, Z_{0}<+\infty$, and $u(x, y, z)$ admits summable fractional derivatives ${ }^{C} D_{x_{0}^{+}}^{1+\alpha},{ }^{C} D_{y_{0}^{+}}^{1+\beta}$ and ${ }^{C} D_{z_{0}^{+}}^{1+\gamma}$. Taking the integral operator $I_{x_{0}^{+}}^{1+\alpha}$ from both sides of (13) and taking into account (4) we get

$$
\begin{aligned}
& u(x, y, z)-u\left(x_{0}, y, z\right)-\left(x-x_{0}\right) u_{x}^{\prime}\left(x_{0}, y, z\right)+\left(I_{x_{0}^{+}}^{1+\alpha C} D_{y_{0}^{+}}^{1+\beta} u\right)(x, y, z)+\left(I_{x_{0}^{+}}^{1+\alpha} D_{z_{0}^{+}}^{1+\gamma} u\right)(x, y, z) \\
& \quad=\lambda\left(I_{x_{0}^{+}}^{1+\alpha} u\right)(x, y, z) .
\end{aligned}
$$

Now, applying the operator $I_{y_{0}^{+}}^{1+\beta}$ to both sides of the previous expression and using Fubini's Theorem we get

$$
\begin{aligned}
& \left(I_{y_{0}^{+}}^{1+\beta} u\right)(x, y, z)-\left(I_{y_{0}^{+}}^{1+\beta} f_{0}\right)(y, z)-\left(x-x_{0}\right)\left(I_{y_{0}^{+}}^{1+\beta} f_{1}\right)(y, z) \\
& \quad+\left(I_{x_{0}^{+}}^{1+\alpha} u\right)(x, y, z)-\left(I_{x_{0}^{+}}^{1+\alpha} u\right)\left(x, y_{0}, z\right)-\left(y-y_{0}\right)\left(I_{x_{0}^{+}}^{1+\alpha} u_{y}^{\prime}\right)\left(x, y_{0}, z\right) \\
& \quad+\left(I_{x_{0}^{+}}^{1+\alpha} I_{y_{0}^{+}}^{1+\beta C} D_{z_{0}^{+}}^{1+\gamma} u\right)(x, y, z)=\lambda\left(I_{x_{0}^{+}}^{1+\alpha} I_{y_{0}^{+}}^{1+\beta} u\right)(x, y, z)
\end{aligned}
$$


where we denote the Cauchy conditions by

$$
f_{0}(y, z)=u\left(x_{0}, y, z\right), \quad f_{1}(y, z)=u_{x}^{\prime}\left(x_{0}, y, z\right) .
$$

Finally, applying the operator $I_{z_{0}^{+}}^{1+\gamma}$ to both sides of equation 15 and using Fubini's Theorem we get

$$
\begin{aligned}
& \left(I_{y_{0}^{+}}^{1+\beta} I_{z_{0}^{+}}^{1+\gamma} u\right)(x, y, z)-\left(I_{y_{0}^{+}}^{1+\beta} I_{z_{0}^{+}}^{1+\gamma} f_{0}\right)(y, z)-\left(x-x_{0}\right)\left(I_{y_{0}^{+}}^{1+\beta} I_{z_{0}^{+}}^{1+\gamma} f_{1}\right)(y, z) \\
& \quad+\left(I_{x_{0}^{+}}^{1+\alpha} I_{z_{0}^{+}}^{1+\gamma} u\right)(x, y, z)-\left(I_{x_{0}^{+}}^{1+\alpha} I_{z_{0}^{+}}^{1+\gamma} h_{0}\right)(x, z)-\left(y-y_{0}\right)\left(I_{x_{0}^{+}}^{1+\alpha} I_{z_{0}^{+}}^{1+\gamma} h_{1}\right)(x, z) \\
& \quad+\left(I_{x_{0}^{+}}^{1+\alpha} I_{y_{0}^{+}}^{1+\beta} u\right)(x, y, z)-\left(I_{x_{0}^{+}}^{1+\alpha} I_{y_{0}^{+}}^{1+\beta} u\right)\left(x, y, z_{0}\right)-\left(z-z_{0}\right)\left(I_{x_{0}^{+}}^{1+\alpha} I_{y_{0}^{+}}^{1+\beta} u_{z}^{\prime}\right)\left(x, y, z_{0}\right) \\
& \quad=\lambda\left(I_{x_{0}^{+}}^{1+\alpha} I_{y_{0}^{+}}^{1+\beta} I_{z_{0}^{+}}^{1+\gamma} u\right)(x, y, z),
\end{aligned}
$$

which is equivalent to

$$
\begin{aligned}
& \left(I_{y_{0}^{+}}^{1+\beta} I_{z_{0}^{+}}^{1+\gamma} u\right)(x, y, z)+\left(I_{x_{0}^{+}}^{1+\alpha} I_{z_{0}^{+}}^{1+\gamma} u\right)(x, y, z)+\left(I_{x_{0}^{+}}^{1+\alpha} I_{y_{0}^{+}}^{1+\beta} u\right)(x, y, z)-\lambda\left(I_{x_{0}^{+}}^{1+\alpha} I_{y_{0}^{+}}^{1+\beta} I_{z_{0}^{+}}^{1+\gamma} u\right)(x, y, z) \\
& =\left(I_{y_{0}^{+}}^{1+\beta} I_{z_{0}^{+}}^{1+\gamma} f_{0}\right)(y, z)+\left(x-x_{0}\right)\left(I_{y_{0}^{+}}^{1+\beta} I_{z_{0}^{+}}^{1+\gamma} f_{1}\right)(y, z) \\
& \quad+\left(I_{x_{0}^{+}}^{1+\alpha} I_{z_{0}^{+}}^{1+\gamma} h_{0}\right)(x, z)+\left(y-y_{0}\right)\left(I_{x_{0}^{+}}^{1+\alpha} I_{z_{0}^{+}}^{1+\gamma} h_{1}\right)(x, z) \\
& \quad+\left(I_{x_{0}^{+}}^{1+\alpha} I_{y_{0}^{+}}^{1+\beta} g_{0}\right)(x, y)+\left(z-z_{0}\right)\left(I_{x_{0}^{+}}^{1+\alpha} I_{y_{0}^{+}}^{1+\beta} g_{1}\right)(x, y)
\end{aligned}
$$

where we denote the Cauchy conditions by

$$
\begin{aligned}
h_{0}(x, z) & =u\left(x, y_{0}, z\right), & h_{1}(x, z) & =u_{y}^{\prime}\left(x, y_{0}, z\right), \\
g_{0}(x, y) & =u\left(x, y, z_{0}\right), & g_{1}(x, y) & =u_{z}^{\prime}\left(x, y, z_{0}\right) .
\end{aligned}
$$

We observe that the fractional integrals in (17) are Laplace-transformable functions. Therefore, we may apply the two-dimensional Laplace transform to $y$ and $z$ :

$$
F\left(s_{1}, s_{2}\right)=\mathcal{L}\{f\}\left(s_{1}, s_{2}\right)=\int_{y_{0}}^{+\infty} \int_{z_{0}}^{+\infty} e^{-s_{1} y-s_{2} z} f(y, z) d z d y, \quad \operatorname{Re}\left(s_{1}\right) \geq a_{1}>0, \quad \operatorname{Re}\left(s_{2}\right) \geq a_{2}>0 .
$$

Taking into account its convolution and operational properties [6, 15] we obtain the following relations:

$$
\begin{aligned}
& \mathcal{L}\left\{I_{y_{0}^{+}}^{1+\beta} I_{z_{0}^{+}}^{1+\gamma} u\right\}\left(x, s_{1}, s_{2}\right)=s_{1}^{-1-\beta} s_{2}^{-1-\gamma} \mathcal{U}\left(x, s_{1}, s_{2}\right), \\
& \mathcal{L}\left\{I_{x_{0}^{+}}^{1+\alpha} I_{y_{0}^{+}}^{1+\beta} u\right\}\left(x, s_{1}, s_{2}\right)=\frac{s_{1}^{-1-\beta}}{\Gamma(1+\alpha)} \int_{x_{0}}^{x}(x-t)^{\alpha} \mathcal{U}\left(x, s_{1}, s_{2}\right) d t, \\
& \mathcal{L}\left\{\left(I_{x_{0}^{+}}^{1+\alpha} I_{z_{0}^{+}}^{1+\gamma} h_{0}\right)\right\}\left(x, s_{1}, s_{2}\right)=e^{-y_{0} s_{1}} s_{1}^{-1} s_{2}^{-1-\gamma}\left(I_{x_{0}^{+}}^{1+\alpha} h_{0}\right)\left(x, y_{0}, s_{2}\right), \\
& \mathcal{L}\left\{\left(y-y_{0}\right)\left(I_{x_{0}^{+}}^{1+\alpha} I_{z_{0}^{+}}^{1+\gamma} h_{1}\right)\right\}\left(x, s_{1}, s_{2}\right)=e^{-y_{0} s_{1}} s_{1}^{-2} s_{2}^{-1-\gamma}\left(I_{x_{0}^{+}}^{1+\alpha} h_{1}\right)\left(x, y_{0}, s_{2}\right) .
\end{aligned}
$$

Proceeding in a similar way we obtain the Laplace transform of the remaining terms of (17). Combining all the resulting terms and multiplying by $s_{1}^{1+\beta} s_{2}^{1+\gamma}$ we obtain the following second kind homogeneous integral equation of Volterra type:

$$
\begin{aligned}
& \mathcal{U}\left(x, s_{1}, s_{2}\right)+\frac{\left(s_{1}^{1+\beta}+s_{2}^{1+\gamma}-\lambda\right)}{\Gamma(1+\alpha)} \int_{x_{0}}^{x}(x-t)^{\alpha} \mathcal{U}\left(t, s_{1}, s_{2}\right) d t \\
& \quad=F\left(x, s_{1}, s_{2}\right)+e^{-y_{0} s_{1}}\left(I_{x_{0}^{+}}^{1+\alpha}\left(s_{1}^{\beta} h_{0}+s_{1}^{\beta-1} h_{1}\right)\right)\left(x, s_{1}, s_{2}\right)+e^{-z_{0} s_{2}}\left(I_{x_{0}^{+}}^{1+\alpha}\left(s_{2}^{\gamma} g_{0}+s_{2}^{\gamma-1} g_{1}\right)\right)\left(x, s_{1}, s_{2}\right)
\end{aligned}
$$

where

$$
\begin{aligned}
& F\left(x, s_{1}, s_{2}\right)=F_{0}\left(s_{1}, s_{2}\right)+\left(x-x_{0}\right) F_{1}\left(s_{1}, s_{2}\right) \\
& F_{i}\left(s_{1}, s_{2}\right)=\int_{y_{0}}^{Y_{0}} \int_{z_{0}}^{Z_{0}} f_{i}(y, z) e^{-s_{1} y} e^{-s_{2} z} d z d y, \quad i=0,1 .
\end{aligned}
$$


In Theorem 2.6 considering $\lambda=-\left(s_{1}^{1+\beta}+s_{2}^{1+\gamma}-\lambda\right), f$ the right hand side of [20, and replacing $\alpha$ by $1+\alpha$, we conclude by 12 that the unique solution of 20 is

$$
\begin{aligned}
& \mathcal{U}\left(x, s_{1}, s_{2}\right) \\
& =F\left(x, s_{1}, s_{2}\right)+e^{-y_{0} s_{1}}\left(I_{x_{0}^{+}}^{1+\alpha}\left(s_{1}^{\beta} h_{0}+s_{1}^{\beta-1} h_{1}\right)\right)\left(x, s_{1}, s_{2}\right)+e^{-z_{0} s_{2}}\left(I_{x_{0}^{+}}^{1+\alpha}\left(s_{2}^{\gamma} g_{0}+s_{2}^{\gamma-1} g_{1}\right)\right)\left(x, s_{1}, s_{2}\right) \\
& \quad-\left(s_{1}^{1+\beta}+s_{2}^{1+\gamma}-\lambda\right) \int_{x_{0}}^{x}(x-t)^{\alpha} E_{1+\alpha, 1+\alpha}\left(-\left(s_{1}^{1+\beta}+s_{2}^{1+\gamma}-\lambda\right)(x-t)^{1+\alpha}\right) \\
& \quad \times\left(F\left(t, s_{1}, s_{2}\right)+e^{-y_{0} s_{1}}\left(I_{x_{0}^{+}}^{1+\alpha}\left(s_{1}^{\beta} h_{0}+s_{1}^{\beta-1} h_{1}\right)\right)\left(t, s_{1}, s_{2}\right)+e^{-z_{0} s_{2}}\left(I_{x_{0}^{+}}^{1+\alpha}\left(s_{2}^{\gamma} g_{0}+s_{2}^{\gamma-1} g_{1}\right)\right)\left(t, s_{1}, s_{2}\right)\right) d t,
\end{aligned}
$$

which involves as the kernel the two-parameter Mittag-Leffler function. In order to interchange the integral with the series that comes from the Mittag-Leffler function we are going to study the absolute convergence. Thus, we have:

$$
\begin{aligned}
& \mid\left(s_{1}^{1+\beta}+s_{2}^{1+\gamma}-\lambda\right) \int_{x_{0}}^{x}(x-t)^{\alpha} E_{1+\alpha, 1+\alpha}\left(-\left(s_{1}^{1+\beta}+s_{2}^{1+\gamma}-\lambda\right)(x-t)^{1+\alpha}\right) \\
& \times\left(F\left(t, s_{1}, s_{2}\right)+e^{-y_{0} s_{1}}\left(I_{x_{0}^{+}}^{1+\alpha}\left(s_{1}^{\beta} h_{0}+s_{1}^{\beta-1} h_{1}\right)\right)\left(t, s_{1}, s_{2}\right)+e^{-z_{0} s_{2}}\left(I_{x_{0}^{+}}^{1+\alpha}\left(s_{2}^{\gamma} g_{0}+s_{2}^{\gamma-1} g_{1}\right)\right)\left(t, s_{1}, s_{2}\right)\right) d t \mid \\
& \leq\left|F_{0}\left(s_{1}, s_{2}\right)\right| \sum_{n=0}^{+\infty} \frac{\left(\left|s_{1}\right|^{1+\beta}+\left|s_{2}\right|^{1+\gamma}+|\lambda|\right)^{n+1}}{\Gamma((n+1)(1+\alpha))} \int_{x_{0}}^{x}(x-t)^{n(1+\alpha)+\alpha} d t \\
& +\left|F_{1}\left(s_{1}, s_{2}\right)\right| \sum_{n=0}^{+\infty} \frac{\left(\left|s_{1}\right|^{1+\beta}+\left|s_{2}\right|^{1+\gamma}+|\lambda|\right)^{n+1}}{\Gamma((n+1)(1+\alpha))} \int_{x_{0}}^{x}(x-t)^{n(1+\alpha)+\alpha}\left(t-x_{0}\right) d t \\
& +e^{-y_{0} s_{1}} \sum_{n=0}^{+\infty}\left\{\frac{\left(\left|s_{1}\right|^{1+\beta}+\left|s_{2}\right|^{1+\gamma}+|\lambda|\right)^{n+1}}{\Gamma((n+1)(1+\alpha))}\right. \\
& \left.\int_{x_{0}}^{x}(x-t)^{n(1+\alpha)+\alpha} \frac{1}{\Gamma(1+\alpha)} \int_{x_{0}}^{t}(t-w)^{\alpha}\left|s_{1}^{\beta} h_{0}\left(w, y_{0}, s_{2}\right)+s_{1}^{\beta-1} h_{1}\left(w, y_{0}, s_{2}\right)\right| d w d t\right\} \\
& +e^{-z_{0} s_{2}} \sum_{n=0}^{+\infty}\left\{\frac{\left(\left|s_{1}\right|^{1+\beta}+\left|s_{2}\right|^{1+\gamma}+|\lambda|\right)^{n+1}}{\Gamma((n+1)(1+\alpha))}\right. \\
& \left.\int_{x_{0}}^{x}(x-t)^{n(1+\alpha)+\alpha} \frac{1}{\Gamma(1+\alpha)} \int_{x_{0}}^{t}(t-w)^{\alpha}\left|s_{2}^{\gamma} g_{0}\left(w, s_{1}, z_{0}\right)+s_{2}^{\gamma-1} g_{1}\left(w, s_{1}, z_{0}\right)\right| d w d t\right\} \text {. }
\end{aligned}
$$

Taking into account the definition of the functions $\sqrt{18}, \sqrt{19}$ and the fact that $u$ admits summable fractional derivatives, we can guarantee the existence of constants $M_{1}, M_{2}, M_{3}, M_{4} \in \mathbb{R}^{+}$such that

$$
\begin{aligned}
& \left|s_{1}^{\beta} h_{0}\left(x, y_{0}, s_{2}\right)+s_{1}^{\beta-1} h_{1}\left(x, y_{0}, s_{2}\right)\right| \leq\left|s_{1}\right|^{\beta} M_{1}+\left|s_{1}\right|^{\beta-1} M_{2}, \quad \forall x \in\left[x_{0}, X_{0}\right], \quad \forall \operatorname{Re}\left(s_{2}\right) \geq a_{2} \\
& \left|s_{2}^{\gamma} g_{0}\left(x, s_{1}, z_{0}\right)+s_{2}^{\gamma-1} g_{1}\left(x, s_{1}, z_{0}\right)\right| \leq\left|s_{2}\right|^{\gamma} M_{3}+\left|s_{2}\right|^{\gamma-1} M_{4}, \quad \forall x \in\left[x_{0}, X_{0}\right], \quad \forall \operatorname{Re}\left(s_{1}\right) \geq a_{1} .
\end{aligned}
$$

Therefore, 23 becomes less or equal than

$$
\begin{aligned}
& \mathbf{A}\left(X_{0}-x_{0}\right)^{1+\alpha} E_{1+\alpha, 2+\alpha}\left(\mathbf{A}\left(X_{0}-x_{0}\right)^{1+\alpha}\right) \int_{y_{0}}^{Y_{0}} \int_{z_{0}}^{Z_{0}}\left|f_{0}(y, z)\right| e^{-a_{1} y} e^{-a_{2} z} d z d y \\
& +\mathbf{A}\left(X_{0}-x_{0}\right)^{2+\alpha} E_{1+\alpha, 3+\alpha}\left(\mathbf{A}\left(X_{0}-x_{0}\right)^{1+\alpha}\right) \int_{y_{0}}^{Y_{0}} \int_{z_{0}}^{Z_{0}}\left|f_{1}(y, z)\right| e^{-a_{1} y} e^{-a_{2} z} d z d y \\
& +\frac{\mathbf{A}}{1+\alpha}\left(X_{0}-x_{0}\right)^{2(1+\alpha)}\left[e^{-y_{0} s_{1}}\left(\left|s_{1}\right|^{\beta} M_{1}+\left|s_{1}\right|^{\beta-1} M_{2}\right)+e^{-z_{0} s_{2}}\left(\left|s_{2}\right|^{\gamma} M_{3}+\left|s_{2}\right|^{\gamma-1} M_{4}\right)\right] \\
& \left.\quad \times{ }_{2} \Psi_{2}\left[\begin{array}{c}
(1,1) ;(1,1) \\
(2,1) ;(3+2 \alpha, 1+\alpha)
\end{array}\right], \mathbf{A}\left(X_{0}-x_{0}\right)^{1+\alpha}\right]
\end{aligned}
$$

where $\mathbf{A}=\left|s_{1}\right|^{1+\beta}+\left|s_{2}\right|^{1+\gamma}+|\lambda|$ and ${ }_{p} \Psi_{q}$ is the Fox-Wright function (see [19]). From the properties of the Mittag-Leffler and Fox-Wright functions we conclude that 24) is finite and, therefore, we can interchange the integral with the series. After straightforward calculations we obtain the following auxiliar result. 
Lemma 3.1 The unique solution of the homogeneous integral Volterra equation (20) in the class of summable functions is given by

$$
\begin{aligned}
& \mathcal{U}\left(x, s_{1}, s_{2}\right) \\
& =E_{1+\alpha, 1}\left(-\left(s_{1}^{1+\beta}+s_{2}^{1+\gamma}-\lambda\right)\left(x-x_{0}\right)^{1+\alpha}\right) F_{0}\left(s_{1}, s_{2}\right) \\
& \quad+\left(x-x_{0}\right) E_{1+\alpha, 2}\left(-\left(s_{1}^{1+\beta}+s_{2}^{1+\gamma}-\lambda\right)\left(x-x_{0}\right)^{1+\alpha}\right) F_{1}\left(s_{1}, s_{2}\right) \\
& \quad+e^{-y_{0} s_{1}} \sum_{n=0}^{+\infty}(-1)^{n}\left(s_{1}^{1+\beta}+s_{2}^{1+\gamma}-\lambda\right)^{n}\left(I_{x_{0}^{+}}^{(1+\alpha)(n+1)}\left(s_{1}^{\beta} h_{0}+s_{1}^{\beta-1} h_{1}\right)\right)\left(x, s_{1}, s_{2}\right) \\
& \quad+e^{-z_{0} s_{2}} \sum_{n=0}^{+\infty}(-1)^{n}\left(s_{1}^{1+\beta}+s_{2}^{1+\gamma}-\lambda\right)^{n}\left(I_{x_{0}^{+}}^{(1+\alpha)(n+1)}\left(s_{2}^{\gamma} g_{0}+s_{2}^{\gamma-1} g_{1}\right)\right)\left(x, s_{1}, s_{2}\right), l
\end{aligned}
$$

where $F_{0}, F_{1}, h_{0}, h_{1}, g_{0}, g_{1}$ are given by (21), (18), and (19).

In order to cancel the Laplace transform we need to take into account its distributional form in Zemanian's space (for more details about generalized integral transforms see [23). Since $n(1+\beta)=[n(1+\beta)]+\{n(1+\beta)\}$ where $\{\cdot\}$ denotes the fractional part of the number and using the relations $\mathcal{L}^{-1}\left\{s^{\nu} F(s)\right\}=\left(I_{y_{0}^{+}}^{-\nu} f\right)(y)$ for $\operatorname{Re}(\nu)<0$ and $\mathcal{L}^{-1}\left\{s^{k} F_{i}(s)\right\}=f_{i}^{(k)}(y)$ for $k \in \mathbb{N}$ in distributional sense (see [23]), we obtain the following relation in the one-dimensional case:

$$
\begin{aligned}
\mathcal{L}^{-1}\left\{s^{n(1+\beta)} F(s)\right\}(y) & =\lim _{r \rightarrow+\infty} \int_{\sigma-i r}^{\sigma+i r} s^{n(1+\beta)} F(s) e^{s y} d s \\
& =\lim _{r \rightarrow+\infty} \int_{\sigma-i r}^{\sigma+i r} s^{n(1+\beta)-1} s^{[n(1+\beta)]+1} F(s) e^{s y} d s \\
& =\left(I_{y_{0}^{+}}^{1-\{n(1+\beta)\}} f^{([n(1+\beta)]+1)}\right)(y) \\
& =\left({ }^{C} D_{y_{0}^{+}}^{n(1+\beta)} f\right)(y),
\end{aligned}
$$

with $\mathcal{L}^{-1}\{F(s)\}=f(y)$. The previous relation can be generalized, via straightforward calculations, to the two dimensional case, yielding the following auxiliar lemma.

Lemma 3.2 The following relation holds

$$
\lim _{r_{1}, r_{2} \rightarrow+\infty} \int_{\sigma_{1}-i r_{1}}^{\sigma_{1}+i r_{1}} \int_{\sigma_{2}-i r_{2}}^{\sigma_{2}+i r_{2}} s_{1}^{n(1+\beta)} s_{2}^{n(1+\gamma)} F\left(s_{1}, s_{2}\right) e^{s_{1} y+s_{2} z} d s_{1} d s_{2}=\left({ }^{C} D_{y_{0}^{+}}^{n(1+\beta)}{ }^{C} D_{z_{0}^{+}}^{n(1+\gamma)} f\right)(y, z),
$$

with $\mathcal{L}\{f(y, z)\}=F\left(s_{1}, s_{2}\right)$. Moreover, since $\mathcal{L}^{-1}\left\{s^{k} e^{-s y_{0}}\right\}=\delta_{y_{0}}^{(k)}(y)$, for $k \in \mathbb{N}$, where $\delta$ is Dirac's delta function and the convergence is in $\mathcal{D}^{\prime}$, we obtain in each coordinate

$$
\begin{aligned}
& \lim _{r_{1} \rightarrow+\infty} \int_{\sigma_{1}-i r_{1}}^{\sigma_{1}+i r_{1}} s_{1}^{n(1+\beta)} e^{s_{1}\left(y-y_{0}\right)} d s_{1}=\left({ }^{C} D_{y_{0}^{+}}^{n(1+\beta)} \delta_{y_{0}}\right)(y), \\
& \lim _{r_{2} \rightarrow+\infty} \int_{\sigma_{2}-i r_{2}}^{\sigma_{2}+i r_{2}} s_{2}^{n(1+\gamma)} e^{s_{2}\left(z-z_{0}\right)} d s_{2}=\left({ }^{C} D_{z_{0}^{+}}^{n(1+\gamma)} \delta_{z_{0}}\right)(z) .
\end{aligned}
$$

Therefore, applying the multinomial theorem and Lemma 3.2 to cancel the Laplace transform we get after straightforward calculations the following family of eigenfunctions of (13)

$$
\begin{aligned}
u_{\lambda}(x, y, z)= & \sum_{n=0}^{+\infty}(-1)^{n} \frac{\left(x-x_{0}\right)^{n(1+\alpha)}}{\Gamma((1+\alpha) n+1)}\left({ }^{C} D_{y_{0}^{+}}^{1+\beta}+{ }^{C} D_{z_{0}^{+}}^{1+\gamma}-\lambda\right)^{n} f_{0}(y, z) \\
+ & \sum_{n=0}^{+\infty}(-1)^{n} \frac{\left(x-x_{0}\right)^{n(1+\alpha)+1}}{\Gamma((1+\alpha) n+2)}\left({ }^{C} D_{y_{0}^{+}}^{1+\beta}+{ }^{C} D_{z_{0}^{+}}^{1+\gamma}-\lambda\right)^{n} f_{1}(y, z) \\
+ & \int_{x_{0}}^{x} \sum_{n=0}^{+\infty}(-1)^{n} \frac{(x-t)^{n(1+\alpha)+\alpha}}{\Gamma((1+\alpha) n+1+\alpha)}\left({ }^{C} D_{y_{0}^{+}}^{1+\beta}+{ }^{C} D_{z_{0}^{+}}^{1+\gamma}-\lambda\right)^{n} \\
& \quad\left(\left({ }^{C} D_{y_{0}^{+}}^{\beta} \delta_{y_{0}}\right)(y) h_{0}(t, z)+\left({ }^{C} D_{y_{0}^{+}}^{\beta-1} \delta_{y_{0}}\right)(y) h_{1}(t, z)\right. \\
& \left.+\left({ }^{C} D_{z_{0}^{+}}^{\gamma} \delta_{z_{0}}\right)(z) g_{0}(t, y)+\left({ }^{C} D_{z_{0}^{+}}^{\gamma-1} \delta_{z_{0}}\right)(z) g_{1}(t, y)\right) d t
\end{aligned}
$$


where the convergence of the series is in $\mathcal{D}^{\prime}$. Due to uniform convergence it is possible to interchange the integral with the series and rewrite the last term of (26) as a series of fractional integrals. From the previous calculations we obtain the following theorem describing the eigenfunctions in an operational form using the Mittag-Leffler function $(9)$.

Theorem 3.3 The eigenfunctions of the fractional Laplace operator ${ }^{C} \Delta_{+}^{(\alpha, \beta, \gamma)}$ are given in the operational form using the Mittag-Leffler function by:

$$
\begin{aligned}
u_{\lambda}(x, y, z)= & E_{1+\alpha, 1}\left(-\left(x-x_{0}\right)^{1+\alpha}\left({ }^{C} D_{y_{0}^{+}}^{1+\beta}+{ }^{C} D_{z_{0}^{+}}^{1+\gamma}-\lambda\right)\right) f_{0}(y, z) \\
+ & \left(x-x_{0}\right) E_{1+\alpha, 2}\left(-\left(x-x_{0}\right)^{1+\alpha}\left({ }^{C} D_{y_{0}^{+}}^{1+\beta}+{ }^{C} D_{z_{0}^{+}}^{1+\gamma}-\lambda\right)\right) f_{1}(y, z) \\
+ & \int_{x_{0}}^{x}(x-t)^{\alpha} E_{1+\alpha, 1+\alpha}\left(-(x-t)^{1+\alpha}\left({ }^{C} D_{y_{0}^{+}}^{1+\beta}+{ }^{C} D_{z_{0}^{+}}^{1+\gamma}-\lambda\right)\right) \\
& \left(\left({ }^{C} D_{y_{0}^{+}}^{\beta} \delta_{y_{0}}\right)(y) h_{0}(t, z)+\left({ }^{C} D_{y_{0}^{+}}^{\beta-1} \delta_{y_{0}}\right)(y) h_{1}(t, z)\right. \\
& \left.+\left({ }^{C} D_{z_{0}^{+}}^{\gamma} \delta_{z_{0}}\right)(z) g_{0}(t, y)+\left({ }^{C} D_{z_{0}^{+}}^{\gamma-1} \delta_{z_{0}}\right)(z) g_{1}(t, y)\right) d t
\end{aligned}
$$

where $\lambda \in \mathbb{C}$ and $f_{0}, f_{1}, h_{0}, h_{1}, g_{0}, g_{1}$ are Cauchy's conditions given by (16), (18), and (19).

Proof: We give a direct proof of the theorem. It is based on the fact that ${ }^{C} D_{x_{0}^{+}}^{1+\alpha} 1=0$ and ${ }^{C} D_{x_{0}^{+}}^{1+\alpha}\left(x-x_{0}\right)=0$. We also use the fractional analogous formula for differentiation of integrals depending on a parameter where the upper limit also depends on the same parameter (see [17, Section 2.7.4]). Applying the operator ${ }^{C} \Delta_{+}^{(\alpha, \beta, \gamma)}$ to 26 we get

$$
\begin{aligned}
{ }^{C} \Delta_{+}^{(\alpha, \beta, \gamma)} u_{\lambda}(x, y, z)= & \sum_{n=1}^{+\infty}(-1)^{n} \frac{\left(x-x_{0}\right)^{(n-1)(1+\alpha)}}{\Gamma((1+\alpha) n-\alpha)}\left({ }^{C} D_{y_{0}^{+}}^{1+\beta}+{ }^{C} D_{z_{0}^{+}}^{1+\gamma}-\lambda\right)^{n} f_{0}(y, z) \\
& +\sum_{n=1}^{+\infty}(-1)^{n} \frac{\left(x-x_{0}\right)^{n(1+\alpha)-\alpha}}{\Gamma((1+\alpha) n+1-\alpha)}\left({ }^{C} D_{y_{0}^{+}}^{1+\beta}+{ }^{C} D_{z_{0}^{+}}^{1+\gamma}-\lambda\right)^{n} f_{1}(y, z) \\
& +\int_{x_{0}}^{x} \sum_{n=1}^{+\infty}(-1)^{n} \frac{(x-t)^{n(1+\alpha)-1}}{\Gamma((1+\alpha) n)}\left({ }^{C} D_{y_{0}^{+}}^{1+\beta}+{ }^{C} D_{z_{0}^{+}}^{1+\gamma}-\lambda\right)^{n} \\
& \left(\left({ }^{C} D_{y_{0}^{+}}^{\beta} \delta_{y_{0}}\right)(y) h_{0}(t, z)+\left({ }^{C} D_{y_{0}^{+}}^{\beta-1} \delta_{y_{0}}\right)(y) h_{1}(t, z)\right. \\
& \left.+\left({ }^{C} D_{z_{0}^{+}}^{\gamma} \delta_{z_{0}}\right)(z) g_{0}(t, y)+\left({ }^{C} D_{z_{0}^{+}}^{\gamma-1} \delta_{z_{0}}\right)(z) g_{1}(t, y)\right) d t \\
& +\left({ }^{C} D_{y_{0}^{+}}^{1+\beta}+{ }^{C} D_{z_{0}^{+}}^{1+\gamma}\right) u_{\lambda}(x, y, z) .
\end{aligned}
$$

Rearranging the terms of the series we obtain

$$
\begin{aligned}
\Delta_{+}^{(\alpha, \beta, \gamma)} u_{\lambda}(x, y, z) & =-\left({ }^{C} D_{y_{0}^{+}}^{1+\beta}+{ }^{C} D_{z_{0}^{+}}^{1+\gamma}-\lambda\right) u_{\lambda}(x, y, z)+\left({ }^{C} D_{y_{0}^{+}}^{1+\beta}+{ }^{C} D_{z_{0}^{+}}^{1+\gamma}\right) u_{\lambda}(x, y, z) \\
& =\lambda u_{\lambda}(x, y, z)
\end{aligned}
$$

A family of eigenfunctions for the fractional Laplace operator can be obtained considering $\lambda=0$ as the next theorem states.

Theorem 3.4 A family of fundamental solutions of the fractional Laplace operator $\Delta_{+}^{(\alpha, \beta, \gamma)}$ is given by:

$$
\begin{aligned}
u_{0}(x, y, z)= & E_{1+\alpha, 1}\left(-\left(x-x_{0}\right)^{1+\alpha}\left({ }^{C} D_{y_{0}^{+}}^{1+\beta}+{ }^{C} D_{z_{0}^{+}}^{1+\gamma}\right)\right) f_{0}(y, z) \\
+ & \left(x-x_{0}\right) E_{1+\alpha, 2}\left(-\left(x-x_{0}\right)^{1+\alpha}\left({ }^{C} D_{y_{0}^{+}}^{1+\beta}+{ }^{C} D_{z_{0}^{+}}^{1+\gamma}\right)\right) f_{1}(y, z) \\
+ & \int_{x_{0}}^{x}(x-t)^{\alpha} E_{1+\alpha, 1+\alpha}\left(-(x-t)^{1+\alpha}\left({ }^{C} D_{y_{0}^{+}}^{1+\beta}+{ }^{C} D_{z_{0}^{+}}^{1+\gamma}\right)\right) \\
& \left(\left({ }^{C} D_{y_{0}^{+}}^{\beta} \delta_{y_{0}}\right)(y) h_{0}(t, z)+\left({ }^{C} D_{y_{0}^{+}}^{\beta-1} \delta_{y_{0}}\right)(y) h_{1}(t, z)\right. \\
& \left.+\left({ }^{C} D_{z_{0}^{+}}^{\gamma} \delta_{z_{0}}\right)(z) g_{0}(t, y)+\left({ }^{C} D_{z_{0}^{+}}^{\gamma-1} \delta_{z_{0}}\right)(z) g_{1}(t, y)\right) d t
\end{aligned}
$$


where $f_{0}, f_{1}, h_{1}, h_{0}, g_{0}, g_{1}$ are given by (16), 18), and (19).

In a similar way, applying in (17) the two-dimensional Laplace transform with respect to $x$ and $y$ we obtain the following fundamental solutions

$$
\begin{aligned}
v_{0}(x, y, z)= & E_{1+\beta, 1}\left(-\left(y-y_{0}\right)^{1+\beta}\left({ }^{C} D_{x_{0}^{+}}^{1+\alpha}+{ }^{C} D_{z_{0}^{+}}^{1+\gamma}\right)\right) h_{0}(x, z) \\
+ & \left(y-y_{0}\right) E_{1+\beta, 2}\left(-\left(y-y_{0}\right)^{1+\beta}\left({ }^{C} D_{x_{0}^{+}}^{1+\alpha}+{ }^{C} D_{z_{0}^{+}}^{1+\gamma}\right)\right) h_{1}(x, z) \\
+ & \int_{y_{0}}^{y}(y-t)^{\beta} E_{1+\beta, 1+\beta}\left(-(y-t)^{1+\beta}\left({ }^{C} D_{x_{0}^{+}}^{1+\alpha}+{ }^{C} D_{z_{0}^{+}}^{1+\gamma}\right)\right) \\
& \left(\left({ }^{C} D_{x_{0}^{+}}^{\alpha} \delta_{x_{0}}\right)(x) f_{0}(t, z)+\left({ }^{C} D_{x_{0}^{+}}^{\alpha-1} \delta_{x_{0}}\right)(x) f_{1}(t, z)\right. \\
& \left.+\left({ }^{C} D_{z_{0}^{+}}^{\gamma} \delta_{z_{0}}\right)(z) g_{0}(x, t)+\left({ }^{C} D_{z_{0}^{+}}^{\gamma-1} \delta_{z_{0}}\right)(z) g_{1}(x, t)\right) d t .
\end{aligned}
$$

Furthermore, a similar result can be obtained when we apply the two-dimensional Laplace transform with respect to $x$ and $z$.

Remark 3.5 It is possible to obtain from (28) the fundamental solution of the Euclidean Laplace operator when $\alpha=\beta=\gamma=1$. Since the fundamental solution of the Euclidean Laplace operator in $\mathbb{R}^{3}$ is given (up a constant) by $\left(\left(x-x_{0}\right)^{2}+\left(y-y_{0}\right)^{2}+\left(z-z_{0}\right)^{2}\right)^{-\frac{1}{2}}$, which corresponds to the following power series

$$
\sum_{n=0}^{+\infty}(-1)^{n}\left(\begin{array}{c}
n-\frac{1}{2} \\
n
\end{array}\right) \frac{\left(x-x_{0}\right)^{2 n}}{\left(\left(y-y_{0}\right)^{2}+\left(z-z_{0}\right)^{2}\right)^{n+\frac{1}{2}}}
$$

defined for $\left|\frac{\left(x-x_{0}\right)^{2}}{\left(y-y_{0}\right)^{2}+\left(z-z_{0}\right)^{2}}\right|<1$, we have to consider in 28) $\alpha=\beta=\gamma=1, f_{1}, h_{1}, g_{1}, h_{0}$, and $g_{0}$ the null functions, and $f_{0}$ such that

$$
\left(D_{y}^{2}+D_{z}^{2}\right)^{n} f_{0}(y, z)=\left(\begin{array}{c}
n-\frac{1}{2} \\
n
\end{array}\right) \frac{(2 n) !}{\left(\left(y-y_{0}\right)^{2}+\left(z-z_{0}\right)^{2}\right)^{n+\frac{1}{2}}} .
$$

The function $f_{0}(y, z)=\left(\left(y-y_{0}\right)^{2}+\left(z-z_{0}\right)^{2}\right)^{-\frac{1}{2}}$ satisfies 31). To see this we recall that the $n$-th powers of the p-dimensional Euclidean Laplace satisfies (see [2, (1.5)])

$$
\Delta^{n} r^{k}=\frac{2^{2 n} \Gamma\left(\frac{k}{2}+1\right) \Gamma\left(\frac{k+p}{2}\right)}{\Gamma\left(\frac{k}{2}-n+1\right) \Gamma\left(\frac{k+p}{2}-n\right)} r^{k-2 n}
$$

with $r=\|x\|, x \in \mathbb{R}^{p}, n \in \mathbb{N}$. Therefore, for $p=2$ and $k=-1$ we obtain

$$
\Delta^{n} r^{-1}=\frac{2^{2 n} \pi}{\left(\Gamma\left(\frac{1}{2}-n\right)\right)^{2}} r^{-1-2 n}=\frac{\Gamma\left(n+\frac{1}{2}\right)(2 n) !}{n ! \sqrt{\pi}} r^{-1-2 n}=\left(\begin{array}{c}
n-\frac{1}{2} \\
n
\end{array}\right)(2 n) ! r^{-1-2 n}
$$

which leads immediately to 31 for $f_{0}(y, z)=\left(\left(y-y_{0}\right)^{2}+\left(z-z_{0}\right)^{2}\right)^{-\frac{1}{2}}$.

\section{Fundamental solution of the fractional Dirac operator}

Let $\left\{e_{1}, \cdots, e_{d}\right\}$ be the standard basis of the Euclidean vector space in $\mathbb{R}^{d}$. The associated Clifford algebra $\mathbb{R}_{0, d}$ is the free algebra generated by $\mathbb{R}^{d}$ modulo $x^{2}=-\|x\|^{2} e_{0}$, where $x \in \mathbb{R}^{d}$ and $e_{0}$ is the neutral element with respect to the multiplication operation in the Clifford algebra $\mathbb{R}_{0, d}$. The defining relation induces the multiplication rules

$$
e_{i} e_{j}+e_{j} e_{i}=-2 \delta_{i j},
$$

where $\delta_{i j}$ denotes the Kronecker's delta. In particular, $e_{i}^{2}=-1$ for all $i=1, \ldots, d$. The standard basis vectors thus operate as imaginary units. A vector space basis for $\mathbb{R}_{0, d}$ is given by the set $\left\{e_{A}: A \subseteq\{1, \ldots, d\}\right\}$ with $e_{A}=e_{l_{1}} e_{l_{2}} \ldots e_{l_{r}}$, where $1 \leq l_{1}<\ldots<l_{r} \leq d, 0 \leq r \leq d, e_{\emptyset}:=e_{0}:=1$. Each $a \in \mathbb{R}_{0, d}$ can be written in the 
form $a=\sum_{A} a_{A} e_{A}$, with $a_{A} \in \mathbb{R}$. The conjugation in the Clifford algebra $\mathbb{R}_{0, d}$ is defined by $\bar{a}=\sum_{A} a_{A} \bar{e}_{A}$, where $\bar{e}_{A}=\bar{e}_{l_{r}} \bar{e}_{l_{r-1}} \ldots \bar{e}_{l_{1}}$, and $\bar{e}_{j}=-e_{j}$ for $j=1, \ldots, d, \bar{e}_{0}=e_{0}=1$. An important subspace of the real Clifford algebra $\mathbb{R}_{0, d}$ is the so-called space of paravectors $\mathbb{R}_{1}^{d}=\mathbb{R} \bigoplus \mathbb{R}^{d}$, being the sum of scalars and vectors. Each non-zero vector $a \in \mathbb{R}_{1}^{d}$ has a multiplicative inverse given by $\frac{\bar{a}}{\|a\|^{2}}$.

A $\mathbb{R}_{0, d}$-valued function $f$ over $\Omega \subseteq \mathbb{R}_{1}^{d}$ has the representation $f=\sum_{A} e_{A} f_{A}$, with components $f_{A}: \Omega \rightarrow$ $\mathbb{R}_{0, d}$. Properties such as continuity or differentiability have to be understood componentwise. Next, we recall the Euclidean Dirac operator $D=\sum_{j=1}^{d} e_{j} \partial_{x_{j}}$ and the correspondent conjugate operator $\bar{D}=\sum_{j=1}^{d} \bar{e}_{j} \partial_{x_{j}}$. These operators verify $\bar{D} D=\Delta$, where $\Delta$ is the $d$-dimensional Euclidean Laplace. An $\mathbb{R}_{0, d}$-valued function $f$ is called left-monogenic if it satisfies $D u=0$ on $\Omega$ (resp. right-monogenic if it satisfies $u D=0$ on $\Omega$ ).

For more details about Clifford algebras and basic concepts of its associated function theory we refer the interested reader for example to [4].

In this section we compute the fundamental solution for the three dimensional fractional left Dirac operator defined via Caputo derivatives

$$
\left.\left.{ }^{C} D_{+}^{(\alpha, \beta, \gamma)}:=e_{1}{ }^{C} D_{x_{0}^{+}}^{\frac{1+\alpha}{2}}+e_{2}{ }^{C} D_{y_{0}^{+}}^{\frac{1+\beta}{2}}+e_{3}{ }^{C} D_{z_{0}^{+}}^{\frac{1+\gamma}{2}}, \quad(\alpha, \beta, \gamma) \in\right] 0,1\right]^{3} .
$$

This operator factorizes the fractional Laplace operator ${ }^{C} \Delta_{+}^{(\alpha, \beta, \gamma)}$ for Clifford valued functions $f$ given by $f(x, y, z)=\sum_{A} e_{A} f_{A}(x, y, z)$, where $e_{A} \in\left\{1, e_{1}, e_{2}, e_{3}, e_{1} e_{2}, e_{1} e_{3}, e_{2} e_{3}, e_{1} e_{2} e_{3}\right\}$, and each real valued function $f_{A}$ satisfies one of the sufficient conditions presented in Theorems 2.3, 2.4 or 2.5. In fact, for such functions we can apply the semigroup property (5) to obtain

$$
{ }^{C} D_{x_{0}^{+}}^{\frac{1+\alpha}{2}}\left({ }^{C} D_{x_{0}^{+}}^{\frac{1+\alpha}{2}} f_{A}\right)={ }^{C} D_{x_{0}^{+}}^{1+\alpha} f_{A}, \quad{ }^{C} D_{y_{0}^{+}}^{\frac{1+\beta}{2}}\left({ }^{C} D_{y_{0}^{+}}^{\frac{1+\beta}{2}} f_{A}\right)={ }^{C} D_{y_{0}^{+}}^{1+\beta} f_{A}, \quad{ }^{C} D_{z_{0}^{+}}^{\frac{1+\gamma}{2}}\left({ }^{C} D_{z_{0}^{+}}^{\frac{1+\gamma}{2}} f_{A}\right)={ }^{C} D_{z_{0}^{+}}^{1+\gamma} f_{A} .
$$

Moreover, for the mixed fractional derivatives ${ }^{C} D_{x_{0}^{+}}^{\frac{1+\alpha}{2}}\left({ }^{C} D_{y_{0}^{+}}^{\frac{1+\beta}{2}} f_{A}\right)$, due to the Leibniz's rule for the differentiation under integral sign, Fubini's Theorem and Schwarz's Theorem, we have

$$
\begin{aligned}
& { }^{C} D_{x_{0}^{+}}^{\frac{1+\alpha}{+}}\left({ }^{C} D_{y_{0}^{\frac{1+\beta}{+}}}^{\frac{1+\beta}{2}} f_{A}\right)={ }^{C} D_{y_{0}^{+}}^{\frac{1+\beta}{+}}\left({ }^{C} D_{x_{0}^{+}}^{\frac{1+\alpha}{+}} f_{A}\right) \\
& { }^{C} D_{x_{0}^{+}}^{\frac{1+\alpha}{2}}\left({ }^{C} D_{z_{0}^{+}}^{\frac{1+\gamma}{2}} f_{A}\right)={ }^{C} D_{z_{0}^{+}}^{\frac{1+\gamma}{2}}\left({ }^{C} D_{x_{0}^{+}}^{\frac{1+\alpha}{2}} f_{A}\right) \\
& { }^{C} D_{y_{0}^{\frac{1+\beta}{+}}}\left({ }^{C} D_{z_{0}^{+}}^{\frac{1+\gamma}{+}} f_{A}\right)={ }^{C} D_{z_{0}^{+}}^{\frac{1+\gamma}{+}}\left({ }^{C} D_{y_{0}^{+}}^{\frac{1+\beta}{+}} f_{A}\right) .
\end{aligned}
$$

From (35), (36), 37), (38) and the multiplication rules (33) of the Clifford algebra, we finally get

$$
\overline{{ }^{C} D_{+}^{(\alpha, \beta, \gamma)}}\left({ }^{C} D_{+}^{(\alpha, \beta, \gamma)} f\right)={ }^{C} \Delta_{+}^{(\alpha, \beta, \gamma)} f,
$$

i.e., the fractional Dirac operator factorizes the fractional Laplace operator. In order to get the fundamental solution of ${ }^{C} D_{+}^{(\alpha, \beta, \gamma)}$ we apply this operator to the fundamental solution 28 . In the following theorem, to compute ${ }^{C} D_{x_{0}^{+}}^{\frac{1+\alpha}{2}} u$ we make use of the derivation rule 11 and the fractional analogous formula for differentiation of integrals depending on a parameter where the upper limit also depends on the same parameter (see 17, Section 2.7.4]).

Theorem 4.1 A family of fundamental solutions for the fractional Dirac operator ${ }^{C} D_{+}^{(\alpha, \beta, \gamma)}$ is given by

$$
U_{0}(x, y, z)=e_{1} U_{1}(x, y, z)+e_{2} U_{2}(x, y, z)+e_{3} U_{3}(x, y, z),
$$


where

$$
\begin{aligned}
& U_{1}(x, y, z)=\left({ }^{C} D_{x_{0}^{+}}^{\frac{1+\alpha}{2}} u_{0}\right)(x, y, z) \\
& =\left(x-x_{0}\right)^{-\frac{1+\alpha}{2}} E_{1+\alpha, \frac{1-\alpha}{2}}\left(-\left(x-x_{0}\right)^{1+\alpha}\left({ }^{C} D_{y_{0}^{+}}^{1+\beta}+{ }^{C} D_{z_{0}^{+}}^{1+\gamma}\right)\right) f_{0}(y, z) \\
& +\left(x-x_{0}\right)^{\frac{1-\alpha}{2}} E_{1+\alpha, \frac{3-\alpha}{2}}\left(-\left(x-x_{0}\right)^{1+\alpha}\left({ }^{C} D_{y_{0}^{+}}^{1+\beta}+{ }^{C} D_{z_{0}^{+}}^{1+\gamma}\right)\right) f_{1}(y, z) \\
& +\int_{x_{0}}^{x}(x-t)^{\frac{\alpha-1}{2}} E_{1+\alpha, \frac{1+\alpha}{2}}\left(-(x-t)^{1+\alpha}\left({ }^{C} D_{y_{0}^{+}}^{1+\beta}+{ }^{C} D_{z_{0}^{+}}^{1+\gamma}\right)\right) \\
& \left(\left({ }^{C} D_{y_{0}^{+}}^{\beta} \delta_{y_{0}}\right)(y) h_{0}(t, z)+\left({ }^{C} D_{y_{0}^{+}}^{\beta-1} \delta_{y_{0}}\right)(y) h_{1}(t, z)\right. \\
& \left.+\left({ }^{C} D_{z_{0}^{+}}^{\gamma} \delta_{z_{0}}\right)(z) g_{0}(t, y)+\left({ }^{C} D_{z_{0}^{+}}^{\gamma-1} \delta_{z_{0}}\right)(z) g_{1}(t, y)\right) d t \\
& U_{2}(x, y, z)=\left({ }^{C} D_{y_{0}^{+}}^{\frac{1+\beta}{2}} u_{0}\right)(x, y, z) \\
& =\left(E_{1+\alpha, 1}\left(-\left(x-x_{0}\right)^{1+\alpha}\left({ }^{C} D_{y_{0}^{+}}^{1+\beta}+{ }^{C} D_{z_{0}^{+}}^{1+\gamma}\right)\right){ }^{C} D_{y_{0}^{+}}^{\frac{1+\beta}{2}}\right) f_{0}(y, z) \\
& +\left(x-x_{0}\right)\left(E_{1+\alpha, 2}\left(-\left(x-x_{0}\right)^{1+\alpha}\left({ }^{C} D_{y_{0}^{+}}^{1+\beta}+{ }^{C} D_{z_{0}^{+}}^{1+\gamma}\right)\right){ }^{C} D_{y_{0}^{+}}^{\frac{1+\beta}{2}}\right) f_{1}(y, z) \\
& +\int_{x_{0}}^{x}(x-t)^{\alpha}\left(E_{1+\alpha, 1+\alpha}\left(-(x-t)^{1+\alpha}\left({ }^{C} D_{y_{0}^{+}}^{1+\beta}+{ }^{C} D_{z_{0}^{+}}^{1+\gamma}\right)\right){ }^{C} D_{y_{0}^{+}}^{\frac{1+\beta}{2}}\right) \\
& \left(\left({ }^{C} D_{y_{0}^{+}}^{\beta} \delta_{y_{0}}\right)(y) h_{0}(t, z)+\left({ }^{C} D_{y_{0}^{+}}^{\beta-1} \delta_{y_{0}}\right)(y) h_{1}(t, z)\right. \\
& \left.+\left({ }^{C} D_{z_{0}^{+}}^{\gamma} \delta_{z_{0}}\right)(z) g_{0}(t, y)+\left({ }^{C} D_{z_{0}^{+}}^{\gamma-1} \delta_{z_{0}}\right)(z) g_{1}(t, y)\right) d t \\
& U_{3}(x, y, z)=\left({ }^{C} D_{y_{0}^{+}}^{\frac{1+\beta}{2}} u_{0}\right)(x, y, z) \\
& =\left(E_{1+\alpha, 1}\left(-\left(x-x_{0}\right)^{1+\alpha}\left({ }^{C} D_{y_{0}^{+}}^{1+\beta}+{ }^{C} D_{z_{0}^{+}}^{1+\gamma}\right)\right){ }^{C} D_{z_{0}^{+}}^{\frac{1+\gamma}{2}}\right) f_{0}(y, z) \\
& +\left(x-x_{0}\right)\left(E_{1+\alpha, 2}\left(-\left(x-x_{0}\right)^{1+\alpha}\left({ }^{C} D_{y_{0}^{+}}^{1+\beta}+{ }^{C} D_{z_{0}^{+}}^{1+\gamma}\right)\right){ }^{C} D_{z_{0}^{+}}^{\frac{1+\gamma}{2}}\right) f_{1}(y, z) \\
& +\int_{x_{0}}^{x}(x-t)^{\alpha}\left(E_{1+\alpha, 1+\alpha}\left(-(x-t)^{1+\alpha}\left({ }^{C} D_{y_{0}^{+}}^{1+\beta}+{ }^{C} D_{z_{0}^{+}}^{1+\gamma}\right)\right){ }^{C} D_{z_{0}^{+}}^{\frac{1+\gamma}{2}}\right) \\
& \left(\left({ }^{C} D_{y_{0}^{+}}^{\beta} \delta_{y_{0}}\right)(y) h_{0}(t, z)+\left({ }^{C} D_{y_{0}^{+}}^{\beta-1} \delta_{y_{0}}\right)(y) h_{1}(t, z)\right. \\
& \left.+\left({ }^{C} D_{z_{0}^{+}}^{\gamma} \delta_{z_{0}}\right)(z) g_{0}(t, y)+\left({ }^{C} D_{z_{0}^{+}}^{\gamma-1} \delta_{z_{0}}\right)(z) g_{1}(t, y)\right) d t .
\end{aligned}
$$

The functions $f_{0}, f_{1}, g_{0}, g_{1}, h_{0}, h_{1}$ are given by (16), (18), and (19).

Remark 4.2 It is possible to obtain from $(40$ ) the fundamental solution for the Euclidean Dirac operator when $\alpha=\beta=\gamma=1$. Indeed, since the fundamental solution of the Euclidean Dirac operator in $\mathbb{R}^{3}$ is given (up to a constant) by

$$
-\frac{\left(x-x_{0}\right) e_{1}+\left(y-y_{0}\right) e_{2}+\left(z-z_{0}\right) e_{3}}{\left(\left(x-x_{0}\right)^{2}+\left(y-y_{0}\right)^{2}+\left(z-z_{0}\right)^{2}\right)^{\frac{3}{2}}},
$$

which corresponds to the following vector power series

$$
\begin{aligned}
& -\left(\sum_{n=0}^{+\infty}(-1)^{n}\left(\begin{array}{c}
n+\frac{1}{2} \\
n
\end{array}\right) \frac{\left(x-x_{0}\right)^{2 n+1}}{\left(\left(y-y_{0}\right)^{2}+\left(z-z_{0}\right)^{2}\right)^{n+\frac{3}{2}}}\right) e_{1} \\
& -\left(\sum_{n=0}^{+\infty}(-1)^{n}\left(\begin{array}{c}
n+\frac{1}{2} \\
n
\end{array}\right) \frac{\left(x-x_{0}\right)^{2 n}\left(y-y_{0}\right)}{\left(\left(y-y_{0}\right)^{2}+\left(z-z_{0}\right)^{2}\right)^{n+\frac{3}{2}}}\right) e_{2} \\
& -\left(\sum_{n=0}^{+\infty}(-1)^{n}\left(\begin{array}{c}
n+\frac{1}{2} \\
n
\end{array}\right) \frac{\left(x-x_{0}\right)^{2 n}\left(z-z_{0}\right)}{\left(\left(y-y_{0}\right)^{2}+\left(z-z_{0}\right)^{2}\right)^{n+\frac{3}{2}}}\right) e_{3},
\end{aligned}
$$


we have to consider in (40) $f_{1}, h_{1}, g_{1}, h_{0}$, and $g_{0}$ the null functions, and $f_{0}$ such that

$$
\begin{aligned}
\left(D_{y}^{2}+D_{z}^{2}\right)^{n+1} f_{0}(y, z) & =\frac{(2 n+1) !\left(\begin{array}{c}
n+\frac{1}{2} \\
n
\end{array}\right)}{\left(\left(y-y_{0}\right)^{2}+\left(z-z_{0}\right)^{2}\right)^{n+\frac{3}{2}}} \\
D_{y}\left(D_{y}^{2}+D_{z}^{2}\right)^{n} f_{0}(y, z) & =-\frac{(2 n) !\left(\begin{array}{c}
n+\frac{1}{2} \\
n
\end{array}\right)\left(y-y_{0}\right)}{\left(\left(y-y_{0}\right)^{2}+\left(z-z_{0}\right)^{2}\right)^{n+\frac{3}{2}}} \\
D_{z}\left(D_{y}^{2}+D_{z}^{2}\right)^{n} f_{0}(y, z) & =-\frac{(2 n) !\left(\begin{array}{c}
n+\frac{1}{2} \\
n
\end{array}\right)\left(z-z_{0}\right)}{\left(\left(y-y_{0}\right)^{2}+\left(z-z_{0}\right)^{2}\right)^{n+\frac{3}{2}}} .
\end{aligned}
$$

The function $f_{0}$ that satisfies the three conditions in (45) is the same function as in case of the Laplace. In fact, from (32) it is not difficult to see that the function $f_{0}(y, z)=\left(\left(y-y_{0}\right)^{2}+\left(z-z_{0}\right)^{2}\right)^{-\frac{1}{2}}$ satisfies the tree conditions 45.

Remark 4.3 As final remarks we would like to point out that if in the beginning of this section we consider $u(x, y, z)$ admitting a summable fractional derivative $\left({ }^{C} D_{x_{0}^{+}}^{1+\alpha} u\right)(x, y, z)$ by $x$ and belonging to $I_{y_{0}^{+}}^{1+\beta}\left(L_{1}\right)$ and $I_{z_{0}^{+}}^{1+\gamma}\left(L_{1}\right)$ by $y$ and $z$, then in the expressions (26), (28), (41), (42), and (43) the last term will not appear due to Theorem 2.1. Moreover, it is possible to apply the method of separations of variables to obtain other families of eigenfunctions and fundamental solutions (see [8]).

Acknowledgement: The authors were supported by Portuguese funds through the CIDMA - Center for Research and Development in Mathematics and Applications, and the Portuguese Foundation for Science and Technology ("FCT-Fundação para a Ciência e a Tecnologia"), within project UID/MAT/ 04106/2013. N. Vieira was also supported by FCT via the FCT Researcher Program 2014 (Ref: IF/00271/2014).

\section{References}

[1] D. Applebaum, Lévy processes: from probability to finance and quantum groups, Notices Am. Math. Soc., 51-No.11, (2004), 1336-1347.

[2] N. Aronszajn, T.M. Creese and L.J. Lipkin, Polyharmonic functions, Oxford Mathematical Monographs, Oxford University Press (Clarendon Press), Oxford, 1983.

[3] L.A. Caffarelli, S. Salsa and L. Silvestre, Regularity estimates for the solution and the free boundary of the obstacle problem for the fractional Laplacian, Invent. Math., 171-No.2, (2008), 425-461.

[4] R. Delanghe, F. Sommen and V. Souček, Clifford algebras and spinor-valued functions. A function theory for the Dirac operator, Mathematics and its Applications-Vol.53, Kluwer Academic Publishers, Dordrecht, 1992.

[5] K. Diethelm, The analysis of fractional differential equations. An application-oriented exposition using differential operators of Caputo type, Lecture Notes in Mathematics, Springer, Berlin, 2004.

[6] V.A. Ditkin and A.P. Prudnikov, Integral transforms and operational calculus, International Series of Monographs in Pure and Applied Mathematics-Vol.78, Pergamon Press, Oxford-London-Edinburgh-New York-Paris-Frankfurt, 1965.

[7] M. Ferreira and N. Vieira, Eigenfunctions and fundamental solutions of the fractional Laplace and Dirac operators: the Riemann-Liouville case, Complex Anal. Oper. Theory, 10-No.5, (2016), 1081-1100.

[8] M. Ferreira and N. Vieira, Eigenfunctions and fundamental solutions of the Caputo fractional Laplace and Dirac operators, Modern Trends in Hypercomplex Analysis, Trends in Mathematics Series, S. Bernstein, U. Kähler, I. Sabadini, F.Sommen (eds.), Birkhäuser, Basel, (2016), 191-202.

[9] M. Garg and P. Manohar, Three-dimensional generalized differential transform method for space-time fractional diffusion equation in two space variables with variable coefficients, Palest. J. Math., 4-No.1, (2015), 127-135. 
[10] R. Gorenflo, A.A. Kilbas, F. Mainardi and S. Rogosin, Mittag-Leffler functions. Theory and applications, Springer Monographs in Mathematics, Springer, Berlin, 2014.

[11] R. Gorenflo, Y. Luchko and M. Stojanovć, Fundamental solution of a distributed order time-fractional diffusion-wave equation as probability density, Fract. Calc. Appl. Anal., 16-No.2, (2013), 297-316.

[12] R. Gorenflo and F. Mainardi, Fractional calculus: integral and differential equations of fractional order, In: Fractals and Fractional Calculus in Continuum Mechanics, Eds: A. Carpinteri and F. Mainardi, Springer Verlag, Wien-New York, (1997), 223-276.

[13] B. Guo, X. Pu and F. Huang, Fractional partial differential equations and their numerical solutions, World Scientific, Hackensack, NJ, 2015.

[14] U. Kähler and N. Vieira, Fractional Clifford analysis, Hypercomplex Analysis: New perspectives and applications, Trends in Mathematics, S. Bernstein, U. Kähler, I. Sabadini and F. Sommen (eds.), Birkhäuser, Basel, (2014), 191-201.

[15] A. Kilbas, H.M. Srivastava and J.J. Trujillo, Theory and applications of fractional differential equations, NorthHolland Mathematics Studies-Vol.204, Elsevier, Amsterdam, 2006.

[16] K.S.Miller and B. Ross, An introduction to the fractional calculus and fractional differential equations, John Wiley \& Sons Inco., New York, 1993.

[17] I. Podlubny, Fractional differential equations. An introduction to fractional derivatives, fractional differential equations, to methods of their solution and some of their applications, Mathematics in Science and Engineering-Vol.198, Academic Press, San Diego, CA, 1999.

[18] S.G. Samko, A.A. Kilbas and O.I. Marichev, Fractional integrals and derivatives: theory and aqpllications, Gordon and Breach, New York, NY, 1993.

[19] H.M. Srivastava and H.L. Manocha, A treatise on generating functions, Ellis Horwood Series in Mathematics and Its Applications, Halsted Press (division of John Wiley \& Sons), Chichester (Ellis Horwood Limited), New York etc., 1984 .

[20] J.L. Vázquez, Recent progress in the theory of nonlinear diffusion with fractional Laplacian operators, Discrete Contin. Dyn. Syst., Ser.7-No.4, (2014), 857-885.

[21] N. Vieira, Fischer Decomposition and Cauchy-Kovalevskaya extension in fractional Clifford analysis: the RiemannLiouville case, Proc. Edinb. Math. Soc., II. Ser., in press, DOI: 10.1017/S0013091516000109.

[22] S. Yakubovich, Eigenfunctions and fundamental solutions of the fractional two-parameter Laplacian, Int. J. Math. Math. Sci., Article ID 541934, (2010), 18p.

[23] A.H. Zemanian, Generalized integral transformations,Pure Applied Mathematics-Vol.18, Interscience Publishers (division of John Wiley \& Sons), New York etc., 1968.

[24] Y. Zhou, Basic theory of fractional differential equations, World Scientific, Hackensack, NJ, 2014. 